\title{
PRIMARY BILIARY CHOLANGITIS IN A PATIENT WITH DIFFUSE SYSTEMIC SCLEROSIS
}

Marília Bulhões Calheiros ${ }^{1, \star}$, Aloma Guinami Scabora ${ }^{1}$, Vani Abreu de Souza Filho ${ }^{1}$, Camille Constanza Codogno Postigo Castroㅇ, Ana Paula Toledo del Rio ${ }^{1}$, Júlia Guimarães Fernandes Costa $^{1}$, Marlone Cunha da Silva ${ }^{1}$, Alisson Aliel Vigano Pugliesi ${ }^{1}$, Marilia de Paula Souza dos Santos $^{1}$, Zoraida Sachetto ${ }^{1}$

1.Universidade Estadual de Campinas, Campinas (SP), Brazil.

*Corresponding author: lilabulhoesc@gmail.com

\section{BACKGROUND}

Systemic sclerosis (SSC) often occurs in association with other autoimmune diseases. Primary biliary cholangitis (PBC) is a liver disease characterized by the destruction of the intrahepatic biliary tract. The association between the two diseases is not uncommon (ranging from 1-20\% in the literature), especially in those patients with limited cutaneous SSc (ICSSC) and with positive anticentromere. Although the CBP is an autoimmune disease, its treatment does not involve the use of immunosuppressive agents.

\section{CASE REPORT}

A 36-year-old female patient is referred to rheumatology outpatient care with a previous diagnosis of rheumatoid arthritis (RA) for evaluation of jaundice and progressive cutaneous thickening. She was diagnosed with diffuse cutaneous SSc (dcSSc) (positive ANA, positive anti-SCl-70, skin thickness, Raynaud's phenomenon, abnormal nailfold capillary pattern -SD pattern) and cholestatic syndrome (expressive elevation of alkaline phosphatase and gamma-glutamyl-transpeptidase). Structural evaluation of the biliary tract by magnetic resonance cholangiopancreatography revealed no alterations, indicating a possible intrahepatic disorder. The subsequent positivity of the antimitochondrial antibody confirmed the diagnosis of PBC and dispensed the need for liver biopsy. She was referred to gastroenterology and started treatment with ursodeoxycholic acid.

\section{CONCLUSION}

Patients with SSc, regardless of classification and autoantibody profile, should be evaluated for the possibility of PBC in the presence of cholestatic jaundice. 